\title{
UNC-55, an Orphan Nuclear Hormone Receptor, Orchestrates Synaptic Specificity among Two Classes of Motor Neurons in Caenorhabditis elegans
}

\author{
H. Mimi Zhou and W. W. Walthall \\ Department of Biology and Center for Neural Communication and Computation, Georgia State University, \\ Atlanta, Georgia 30303
}

Loss of UNC-55 function in the nematode Caenorhabditis elegans causes one motor neuron class, the ventral D (VD) motor neurons, to adopt the synaptic pattern of another motor neuron class, the dorsal D (DD) motor neurons. Here we show that unc-55 encodes a member of the nuclear hormone receptor gene family that is similar to the vertebrate chicken ovalbumin upstream promoter transcription factors. Although the VD and DD motor neuron classes arise from different lineages at differ- ent developmental stages, they share a number of structural and functional features that appear to be the product of identical genetic programs. UNC-55 is expressed in the VD but not the DD motor neurons to modify this genetic program and to create the synaptic pattern that distinguishes the two motor neuron classes from one another.

Key words: synaptic specificity; C. elegans; motor neurons; nuclear hormone receptor; neuromuscular junction; unc-55
The complexity of synaptic patterns present in mature nervous systems raises a fundamental question: how does a finite amount of genetic material create the synaptic diversity present in even the simplest nervous systems? Several lines of evidence suggest that the genetic programs are less diverse than would be predicted by the multitude of synaptic patterns. For example, in the development of layer 5 neurons in the mammalian cortex, visual and motor cortical neurons initially extend projections to the same subcortical regions, including the spinal cord. Subsequently, the structural and functional differences that distinguish these neurons emerge because of differential pruning of collateral branches (O'Leary and Terashima, 1988). A conceptually similar example has been reported in the nematode Caenorhabditis elegans: two sets of mechanosensory neurons initiate either forward or backward locomotion by activating different neural circuits based on whether the animal is touched on the anterior or posterior part of the body (Chalfie and $\mathrm{Au}, 1989$ ). However, the same genetic program regulates the differentiation and function of the two circuits (Way and Chalfie, 1988; Xue et al., 1993). In both examples a common genetic program plus features associated with the relative positions of the neurons cause them to form distinctive synaptic patterns (Chalfie et al., 1983; Walthall and Chalfie, 1988). Our goal is to investigate the mechanisms responsible for

Received Jan. 29, 1998; revised July 6, 1998; accepted Oct. 7, 1998.

This work was supported by the National Science Foundation and a dissertation grant from the Office of Research and Sponsored Programs at Georgia State University. Some of the strains used in this work were supplied by the Caenorhabditis Genetics Center, which is funded by the National Institutes of Health National Center for Research Resources. We thank Mark Borodovsky and Xiaowei Lu for help in computer sequence analysis, Sarah Combs for help in the molecular cloning, and Andrew Fire and colleagues, who provided the gfp cloning vector. We are also grateful to Ann Sluder and Guy Benian for discussions and Sarah Pallas and Vincent Rehder for comments on this manuscript.

Correspondence should be addressed to W. W. Walthall, Department of Biology, Georgia State University, P.O. Box 4010, Atlanta, GA 30303-4010.

Dr. Zhou's present address: Emory University School of Medicine, Department of Gynecology and Obstetrics, Atlanta, GA 30322.

Copyright (C) 1998 Society for Neuroscience $\quad 0270-6474 / 98 / 1810438-07 \$ 05.00 / 0$ modifying the common genetic programs of neurons to create different synaptic specificities.

The precisely defined sets of nerve and muscle cells responsible for locomotion in C. elegans allow genetic and cellular manipulations that provide a unique opportunity for investigating the generation of specific synaptic patterns. The sinuous forward and backward locomotion exhibited by $C$. elegans is produced by two neural circuits; one dedicated to forward movement and the other dedicated to backward movement (Chalfie et al., 1985). These two circuits converge on the dorsal and ventral body wall muscles and two classes of inhibitory motor neurons: 6 dorsal D (DD) motor neurons (born embryonically) and 13 ventral (VD) motor neurons (born postembryonically). In adults, the DD and VD motor neurons form a cross-inhibitory network (Fig. 1A). A common genetic program is responsible for establishing the cellular morphology (Hedgecock et al., 1990; McIntire et al., 1992) and maintaining the neurotransmitter profile (cholinergic inputs and GABAergic outputs) in both classes (McIntire et al., 1993a,b, 1997; Jin et al., 1994). However, none of the identified genes influences the synaptic patterns that distinguish the VD motor neurons from the DD motor neurons. Mutations in the gene unc-55 (uncoordinated) cause the VD motor neurons to adopt the synaptic pattern of the DD motor neurons and result in an asymmetric locomotive pattern when the animals move backward (Walthall and Plunkett, 1995). We report here that unc-55 encodes a nuclear hormone receptor that is expressed in the VD motor neurons but not the DD motor neurons to modify a shared genetic program that produces the synaptic pattern that distinguishes one from the other.

\section{MATERIALS AND METHODS}

Strains and growth of C. elegans. The wild-type strain N2, unc-55 I strains $[j d 3, j d 4, j d 9, j d 9 R$ (revertant), jd13, u411, e402, and e1170] and unc-30 IV (e191) were used. Deficiency strains nDf24 and nDf25 and mutator strains TR679 and RW7000 were used for mapping and cloning of unc-55. C. elegans for general studies were grown on nematode growth medium (NGM)-agar plates as described by Brenner (1974). Strains for DNA preparations were grown on NGM-agarose plates (0.9\% agarose) 
to avoid contaminants that interfere with restriction enzyme digestion. Large numbers of $C$. elegans for obtaining RNA were generated by growing strains in liquid culture. In addition, DNA from a wild-type revertant $(j d 9 R)$ obtained from $u n c-55$ ( $j d 9)$ was examined.

Gene mapping. The unc-55 locus was mapped using standard genetic techniques. The genetic deficiencies $\mathrm{nDf} 24$ and $\mathrm{nDf} 25$ both failed to complement the Unc-55 mutant phenotype. However, nDf25 complements Gld-1 (germline-deficient), whereas $\mathrm{nDf} 24$ does not (Jones and Schedl, 1995), indicating that the breakpoint for nDf24 lies to the right of gld-1 and to the left of unc-55. Three-factor mapping using a transheterozygote unc-13 (e1091) che-3 (e1124)/unc-55 (e402) placed unc-55 to the left of che-3.

Molecular biology. Methods published by Sambrook et al. (1989) were used unless otherwise noted. C. elegans genomic DNA was isolated following published procedures (Emmons et al., 1979). Cosmid DNA was purified using Tip 100 from Qiagen (Chatsworth, CA). DNA transfer, hybridization, and detection used the Genius System and followed protocols in the user's guide from Boehringer Mannheim (Indianapolis, IN).

The $1.8 \mathrm{~kb} P s t \mathrm{I}-E c o \mathrm{RI}$ fragment that contains the upstream regulatory region of unc-55 was cloned from cosmid ZC579 into pUC 18 to create pJD1.8. pJD1.8 was then modified by the addition of a SalI site, and the resulting Pst I-SalI fragment was cloned into pPD95.79, a promoterless green fluorescent protein (gfp) expression vector that was provided by Andrew Fire (Carnegie Institue) (Chalfie et al., 1994). This construct is referred to as $\mathrm{ME} 2$.

$R T-P C R$. Large numbers of mixed stage animals were grown in liquid culture in a fermentor (Bioflo III, New Brunswick Scientific). Total RNA was isolated using Trizol reagent from Life Technologies (Gaithersburg, MD) following the included protocol. mRNA was isolated from total RNA using an Oligotex mRNA kit (Qiagen). Approximately $1.5 \mu \mathrm{g}$ of the isolated mRNA was used in each reverse transcription experiment with $0.5 \mu \mathrm{g}$ of a 15 mer oligo-dT primer. The transcription reaction proceeded at $42^{\circ} \mathrm{C}$ for $1 \mathrm{hr}$ with $1 \times$ first-strand buffer, $8 \mathrm{~mm}$ DTT, $20 \mathrm{U}$ of Rnase inhibitor, $0.8 \mathrm{~mm}$ dNTP, $1 \mu \mathrm{g}$ of nuclease-free BSA, and $20 \mathrm{U}$ of avian myeloblastosis virus reverse transcriptase. One-twentieth of the reversetranscribed DNA was used in each PCR, which was performed under the following conditions in a Perkin-Elmer (Norwalk, CT) thermal cycler: $94^{\circ} \mathrm{C}$ for $1 \mathrm{~min}, 55^{\circ} \mathrm{C}$ for $1 \mathrm{~min}$, and $72^{\circ} \mathrm{C}$ for $3 \mathrm{~min}$, plus cycle elongation of $5 \mathrm{sec}$ for each cycle for a total 30 cycles using regular Taq polymerase. RT-PCR products were then checked on a $1.0 \%$ agarose gel. Primers used to identify unc-55 were mm-1R (ATGGACAGTTCAGTTGTGAAGGAT), TSL-1 (GGTTTAATTACCCAAGTTTGAG), and RT-1 (CTAATTTCTGGAAGGTAGTTG). The unc-55 cDNA was generated by cloning the RT-PCR product into pGEM-T vector from Promega (Madison, WI); the identity of the amplified cDNA was verified by sequencing (automated sequencer; Applied Biosystems, Foster City, CA) and DNA hybridization with an $u n c-55$ probe labeled with digoxygenin.

Transformation in C. elegans. Injection of cosmid DNAs into the gonad (germline) of C. elegans followed the procedures described by Mello et al. (1991). Cosmid F55D12 $(25 \mu \mathrm{g} / \mathrm{ml})$ was injected into the gonad of unc-55 (jd4). A rescued (wild-type locomotion) strain (jdEx1) was obtained from the progeny of the injected animal, and it carries a heritable extrachromosomal array.

Plasmid ME2 $(25 \mu \mathrm{g} / \mathrm{ml})$, which contains the upstream regulatory region of $u n c-55$ fused to a gfp reporter, together with plasmid pRF4 (50 $\mu \mathrm{g} / \mathrm{ml}$ ), a plasmid that contains a $C$. elegans dominant rol-6 mutation (su1006) (Kramer et al., 1990), was injected into the gonad of wild-type unc-55 (N2). Animals with a roller phenotype were isolated from the F1 generation, and their progeny were examined for gfp expression. A strain of roller animals that expressed gfp, $j d E x 2$, was established.

Immunohistochemistry. Animals were processed according to an immunohistochemistry protocol reported by Walthall and Plunkett (1995). The identification of motor neurons in the ventral nerve cord that expressed an unc-55-gfp reporter construct was determined by simultaneously staining for gfp and GABA, a neurotransmitter that is expressed in the ventral nerve cord exclusively in the DD and VD motor neurons, using indirect immunofluorescence. unc-55-gfp was detected using a rabbit anti-gfp antibody (purchased from Clontech, Basingstoke, UK) that was detected with a goat anti-rabbit secondary antibody conjugated to rhodamine. GABA expression was determined using a guinea pig anti-GABA primary antibody (purchased from Protos Biotech Corp., New York, NY) and a goat anti-guinea pig secondary antibody conjugated to fluorescein isothiocyanate (FITC; Sigma, St. Louis, MO). Nuclei were stained by adding Hoechst 33258 to the second of three washes.
Preparations were viewed on a Zeiss (Thornwood, NY) LS 510 laser scanning confocal microscope. To avoid detection of overlapping excitation, scans for rhodamine and FITC fluorescence were run separately. The image stacks presented in Figure 5 were captured and collapsed along the $z$ axis. These captured images were transferred as tagged image files into Adobe (Mountain View, CA) Photoshop, where they were labeled and printed.

\section{RESULTS}

\section{Molecular cloning of the unc-55 gene}

Three-factor mapping with unc-55, che-3, and gld-1 and deficiency tests identified the genetic interval on chromosome I that contains unc-55 (Fig. $2 A$ ). A $5.4 \mathrm{~kb} E c o$ RI probe made from either of two overlapping cosmids in this genetic interval, ZC579 and F55D12, revealed a restriction fragment length polymorphism (RFLP) in three transposon-tagged $u n c-55$ alleles ( $j d 9, j d 13$, and $u 411)$ that corresponded to the size of the C. elegans transposon Tc1 (Fig. 2B,C; Collins et al., 1987).

The cosmid F55D12, which showed RFLPs associated with transposon-tagged unc-55 alleles, was injected into the gonads of unc-55 (jd4) mutants following the procedures of Mello et al. (1991). In transgenic animals backward locomotion was completely restored (Fig. $1 B$ ).

A $1.1 \mathrm{~kb}$ full-length cDNA was isolated by RT-PCR (Fig. 2D). Like many C. elegans genes, unc-55 is trans-spliced at its $5^{\prime}$ end to the RNA splice leader SL1 (Krause and Hirsh, 1987). The SL1derived consensus sequence was found at the $5^{\prime}$ end of the first methionine codon of the unc-55 cDNA. The methionine codon is actually in the context (UUUCAGAUG) that matches the $3^{\prime}$ splice site consensus sequence UUUCAG/G (Blumenthal, 1995). After trans-splicing, the SL1 is fused directly to the $5^{\prime}$ end of AUG in the unc-55 cDNA and creates a putative initiator methionine under a consensus sequence (Kozak, 1991): the A of the AUG is defined as position +1 , and the position -3 is $G$, which comes from the SL1 (Fig. 3). A putative polyadenylation signal, AAUAAA, is located $560 \mathrm{bp}$ downstream of the stop codon TAG (Fig. 2D). No alternative mRNAs were detected by Northern or RT-PCR analyses.

The polymorphic fragments of the three transposon-tagged alleles were sequenced. Each independently isolated allele had the transposon Tc1 inserted into an identical site of $u n c-55$; this site is a consensus target site for the Tc1 transposase (Eide and Anderson, 1988). The insertion site is located in the exon that encodes the DNA-binding domain (DBD) of the protein (Fig. 3).

\section{unc-55 encodes a member of the nuclear hormone receptor superfamily}

The 1113 bp unc-55 cDNA encodes a predicted protein of 371 amino acids (Fig. 3). Comparison of the sequence of UNC-55 with other sequences in various databases indicated that UNC-55 belongs to the superfamily of nuclear hormone receptors and shares particularly high similarity with the chicken ovalbumin upstream promoter transcription factors (COUP-TFs) and the Drosophila seven-up receptors (SVPs) (Fig. 4). The COUP-TFs include the human and mouse apolipoprotein AI regulatory proteins (ARP-1), which are also known as COUP-TF II (Ladias and Karathanasis, 1991), human COUP-TF I (EAR-3 or hCOUP) (Wang et al., 1989), and human- and mouse-related protein EAR-2 (Jonk et al., 1994), and the SVPs include Drosophila steroid receptor seven-up type 1 (SVP-1) and type 2 (Mlodzik et al., 1990) and the zebrafish steroid receptors seven-up type 46 (SVP46) and type 44 (Fjose et al., 1993). 
$\mathbf{A}$

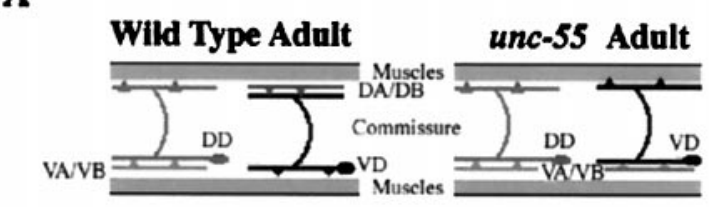

B

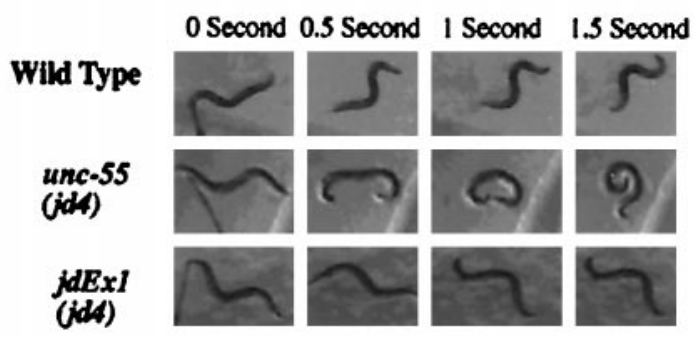

Figure 1. Neural circuit alteration and the resulting uncoordination in unc-55 mutants. $A$, Schmetic diagram of the structures of a VD motor neuron and a DD motor neuron in both a wild-type adult animal and an unc-55 adult mutant. Each VD and DD motor neuron has a ventral cell body (ovals) and processes in the dorsal and ventral nerve cords that are connected via a commissure (the direction of information transfer is indicated by the arrows). In wild-type adults, the VD motor neuron (black) receives synaptic input from the dorsal processes of the excitatory motor neurons $(D A, D B)$ and innervates ventral body wall muscles. The DD motor neuron ( gray) receives synaptic input from the ventral processes of the excitatory motor neurons $(V A, V B)$ and innervates dorsal body wall muscles. In unc-55 adult mutants, the DD and VD motor neurons both have the DD motor neuron synaptic pattern. $B$, The sequence of photographs was taken from video recordings comparing the behavior of an $u n c-55(j d 4)$ mutant with a wild-type and a rescued animal (jdEx1) after a touch with an eyelash on the animal's head. The unc-55 (jd4) animal responded by curling its body from tail to head with the ventral side in the center. The wild-type and rescued animals responded to this stimulus by moving backward sinuously. The interval between frames is $0.5 \mathrm{sec}$. Views are of the left side of the animals with anterior to the left.

Two highly conserved zinc fingers are found near the $\mathrm{N}$ terminus of UNC-55, which based on sequence similarity is the DBD. The DBD contains two conserved elements: the P box (CEGCK) and the D box (RATKN), which are reported to be necessary for sequence-specific DNA binding (Umesono and Evans, 1989; Truss and Beato, 1993) and dimer formation (Glass, 1994), respectively (Fig. 4). A ligand-binding domain (LBD) is located near the $\mathrm{C}$ terminus and contains an LBD-specific signature motif (LBD-SSM), which is reported to be essential for regulatory function (Burke et al., 1996; Downes et al., 1996). Comparison of the amino acid sequence similarity between UNC-55 and hARP-1 is summarized in Table 1. UNC-55 has extremely high similarity (91\%) with hARP-1 in the DBD especially in the P box $(100 \%)$. Although the similarity in the LBD is less $(44 \%)$, UNC-55 possesses highly conserved amino acids in the critical LBD-SSM (90\%).

\section{Identification of ventral cord motor neurons expressing unc-55-gfp}

The temporal and spatial pattern of expression of the unc-55-gfp construct was visualized in the transgenic strain $j d E x 2$ using indirect immunofluorescence visualized using a confocal microscope (Fig. 5). Analysis of six worms revealed a consistent staining pattern in which unc-55-gfp was detected in 2 pairs of neurons in the nerve ring, $\sim 13$ pairs of motor neurons in the ventral nerve cord, and a pair of cells in the preanal ganglion. We have focused

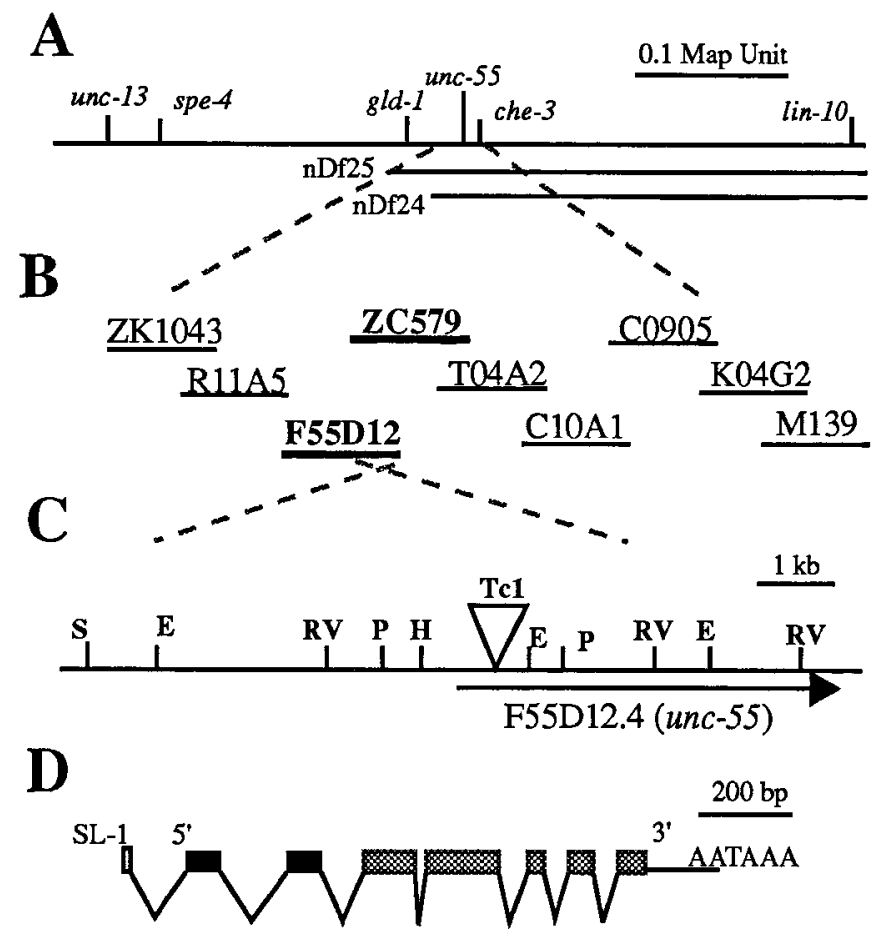

Figure 2. Positional cloning of unc-55. A, Genetic map of the central right arm of chromosome I showing the position of unc-55 in relation to flanking genetic loci. Deficiencies (nDf25 and nDf24) are shown with approximate breakpoints. $B$, Contiguous physical map showing the cosmids between gld-1 and che-3. Line length is not proportional to cosmid size. Cosmids F55D12 and ZC579, which show RFLPs in unc-55 alleles, are indicated in bold face. $C$, Physical map showing the location of unc-55 (arrow) in cosmid F55D12. The fragment shown here has been rotated $180^{\circ}$ from cosmid F55D12 shown in $B$. Restriction sites for HindIII $(H)$, PstI $(P), E c o$ RI $(E), E c o$ RV $(R V)$, and SalI $(S)$ are indicated. The open triangle indicates the approximate site of a transposon-Tc1 insertion in the unc-55(jd9) allele. D, Composite summary of the gene structure as determined from cDNA sequencing. Coding regions are shown in boxes. Trans-splicing occurs at the $5^{\prime}$ end of the transcript to the 22-nucleotide leader SL1 (open box). The putative polyadenylation signal (AATAAA) is indicated in the $3^{\prime}$ end. Regions of sequence similarity, as described in Results, are shown as shaded regions superimposed on the gene structure. The exons encoding the DNA-binding domain are boxed in black, and the exons encoding the ligand-binding domain are boxed in gray.

on the expression in the ventral nerve cord, where we found that unc-55-gfp staining was restricted to the $\mathrm{L} 2$ and $\mathrm{L} 3$ postembryonic stages and occurred in pairs of cells that were usually adjacent to one another. To determine whether any of the stained cells were VD or DD motor neurons, we simultaneousy stained for $u n c-55-g f p$ visualized with rhodamine and the neurotransmitter GABA visualized with FITC. Three populations of neurons were identified, and specific examples of each are indicated in Figure 5. Population 1 was unc-55-gfp(+) and $\mathrm{GABA}(+)$, population 2 was unc-55-gfp(-) and GABA $(+)$, and population 3 was unc-55-gfp $(+)$ and GABA $(-)$. Based on the numbers of neurons in the three classes and the relative positions of the neuron cell bodies visualized with the nuclear stain Hoechst 33258, population 1 corresponds to the VD motor neurons, population 2 corresponds to the DD motor neurons, and population 3 has been tentatively identified as the AS motor neurons. The identification of population 3 as AS is because AS and VD motor neurons are lineal sisters, and as a result the AS motor neurons are positioned anterior and immediately adjacent to the VD motor neurons, which is the location of the unc-55-gfp (+) GABA( $(-)$ cell bodies. 


\begin{tabular}{|c|c|}
\hline & $\begin{array}{r}M Q Q \text { D G S S G A A S I G N S } \\
\text { aagtttgagATGCAGGATGGCTCATCAGGTGCTGCAAGTCTTGGAAACA }\end{array}$ \\
\hline & 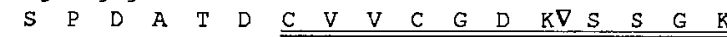 \\
\hline & ITCCCCAGATGCAACAGATTGTGTAGTATGTGGAGATAAATCAAGTGGAA \\
\hline & $\begin{array}{llllllllllllll}G & Q & E & S & C & E & G & C & K & S & F & F & K \\
\end{array}$ \\
\hline & AACATTATGGACAGTTCAGTTGTGAAGGATGCAAAAGTTTCTTCAAAAG \\
\hline & $\begin{array}{llllllllllllllll}S & I & R & R & S & L & S & Y & T & C & R & A & T & K & N & C \\
\end{array}$ \\
\hline & TCAATTCGACGTTCATTATCTTACACGTGTCGTGCTACGAAGAACTGTC \\
\hline & $\begin{array}{lllllllllllllllll}I & D & V & Q & H & R & N & Q & C & Q & Y & C & R & L & T & K \\
\end{array}$ \\
\hline 201 & CATTGACGTGCAÄCACCGAAATCAATGCCAATATTGTCGATTGACAAAA \\
\hline & $\begin{array}{lllll}R & K & E & G & R\end{array}$ \\
\hline 251 & GCATTTCGAATGGGAATGAGAAAAGAAGGAAGAAGATCCAAT GAGACTCAG \\
\hline 38 & 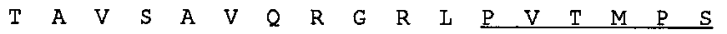 \\
\hline & ACCGCAGTTTCAGCAGTTCAACGAGGTCGTCTACCTGTTACAATGCCAAG \\
\hline 115 & $\begin{array}{lllllllllllllllll}L & E & P & P & N & M & F & L & R & S & P & F & P & F & M & S \\
\end{array}$ \\
\hline 351 & TTTGTTCCCTCCAAATATGTITTTGCGATCTCCATTTCCATTTATGTCTG \\
\hline 13 & $\begin{array}{lllllllllllllllll}P & F & N & P & L & M & T & A & Q & F & T & K & P & S & I & K \\
\end{array}$ \\
\hline 401 & TACCATTCAATCCACTCAT GACTGCTCAATTCACAAAACCATCGATTAAA \\
\hline 180 & $\begin{array}{lllllllllllllllll}E & S & I & F & E & F & A & A & Q & T & I & F & A & T & V & N & W \\
\end{array}$ \\
\hline 45 & GAATCAATATTTGAATTTGCGGCTCAAACAATCTTTGCAACTGTCAACTG \\
\hline 100 & $\begin{array}{llllllllllllllll}A & R & I & S & M & S & N & L & V & K & G & D & Q & L & I & L \\
\end{array}$ \\
\hline & GGCTCGTACAAGTAT GAGTAACCTTGT GAAAGGGGATCAATTGATTCTGC \\
\hline 182 & $\begin{array}{lllll}R & H & S & W & T \\
\end{array}$ \\
\hline 351 & TCCGTCACAGCTGGACGCCCATTTTCATTTTTGCACTGGCTCAATCGAAT \\
\hline & $\begin{array}{llllllllllllllll} & \mathrm{A} & \mathrm{I} & \mathrm{N} & \mathrm{I} & \mathrm{S} & \mathrm{T} & \mathrm{H} & \mathrm{L} & \mathrm{T} & \mathrm{A} & \mathrm{V} & \mathrm{T} & \mathrm{A} & \mathrm{T} & \mathrm{A} \\
\end{array}$ \\
\hline 60 & TTCGCACTCAATCTTTCAACCCATTTAACTGCAGTTACGGCAACGGCGC \\
\hline 215 & $\begin{array}{llllllllllllllll}S & T & E & N & G & S & S & S & L & G & S & K & S & E & D & E\end{array}$ \\
\hline & GTCAACAGAAAATGGATCTTCTTCGTTGGGTTCAAAGTCAGAAGATGAGG \\
\hline 232 & $\begin{array}{lllllll}E & D & E & P & Q & F & Q \\
\end{array}$ \\
\hline 10. & LAGAAAAACCGGARAGAGTATTTGATGAGCCACAGTTTCA \\
\hline & $\begin{array}{lllllllllllllllll}G & F & Q & A & K & I & D & K & I & R & D & F & H & L & D & V \\
\end{array}$ \\
\hline 75. & GGATTTCAGGCGAAAATTGATAAGATCCGTGACTTTCATTTGGATGTCG \\
\hline 264 & $\begin{array}{llllllll}E & S & S & S & I & R & A & V \\
\end{array}$ \\
\hline & TGAAAGCTCGTCTCTTCGAGCGGTTCTTCTTTTTAGTTGCGATGAAGAA \\
\hline 282 & $\begin{array}{lllllllllllllllll}L & E & E & K & G & K & I & E & E & I & V & E & K & L & K & S\end{array}$ \\
\hline 851 & CATTGGAGGAGAAGGGAAAAATTGAAGAAATTGTTGAGAAATT GAAAAG \\
\hline 29 & $\begin{array}{llllllllllllllll}A & V & D & E & Y & C & K & M & N & K & R & S & E & R & Y & H \\
\end{array}$ \\
\hline 90$]$ & GCAGTGGATGAATATTGCAAAATGAATAAACGTAGTGAGAGATATCATC \\
\hline 31. & $\begin{array}{lllllllllllllllll}I & C & E & C & L & Q & L & L & K & S & T & R & N & L & P & I \\
\end{array}$ \\
\hline & AATCTGTGAATGTCTTCAACTTCTCAAATCAACTCGAAATCTTCCAATT \\
\hline 331 & $\begin{array}{llllllllllllllll}R & I & E & F & S & R & L & L & G & I & T & P & L & E & T \\
\end{array}$ \\
\hline & CTCGTCTTTTCTTTTCTCGACTTCTTGGTACAACTCCTCTIGAAAC \\
\hline & $\begin{array}{lllllllllllllll}L & L & I & T & P & P\end{array}$ \\
\hline & TTA \\
\hline & \\
\hline & \\
\hline
\end{tabular}

Figure 3. Nucleotide and predicted protein sequence of the unc-55 cDNA. Nucleotides are numbered beginning with the first of the nine nucleotides (small characters) composing the putative RNA trans-splice leader sequence (SL1). Amino acids are numbered (in italics) beginning with the first methionine. The stop codon is marked by an asterisk. The DNA-binding domain is underlined twice, and the ligand-binding domain is underlined once. A triangle indicates the Tc1 insertion site.

\section{DISCUSSION}

UNC-55 acts at a critical crossroad in the differentiation of the DD and VD motor neurons. Although the two motor neuron classes are generated by distinct lineages, are born at different developmental stages, and exhibit different synaptic specificities, they share a number of morphological and biochemical features that are apparently the product of a single genetic program. This genetic program is initiated in both the DD and VD motor neurons by the expression of unc-30, which encodes a homeodomain-containing protein that is necessary for the morphological and biochemical differentiation of the DD and
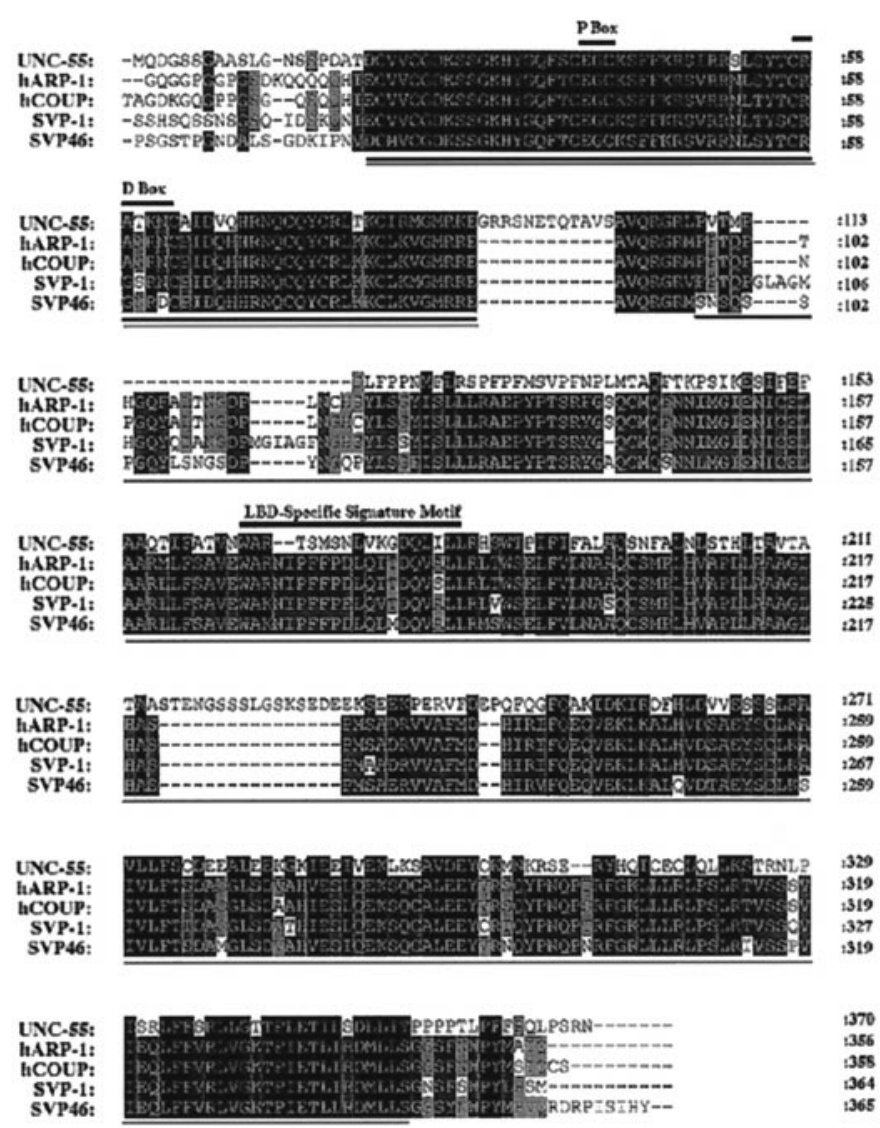

Figure 4. Alignment of UNC-55 with COUP-TFs/SVP homologs. Alignment of UNC-55 with human proteins hARP-1 (Ladias and Karathanasis, 1991), hCOUP (Wang et al., 1989), Drosophila SVP-1 (Mlodzik et al., 1990) and zebrafish SVP46 (Fjose et al., 1993). The first 58 amino acids of hARP1, first 65 amino acids of hCOUP, first 179 amino acids of SVP-1, and first 33 amino acids and last five amino acids of SVP46 are not included. Three or four similar amino acids shared among the five proteins are boxed in gray, and five similar amino acids shared are boxed in black. The following amino acids were considered to be similar: $\mathrm{D}$ and $\mathrm{N}$; A and G; E and Q; S and T; K and R; F, Y, and W; and L, I, $\mathrm{V}$, and M. Gaps are indicated by dashes. The alignment analysis was performed with the CLUSTAL program. The P box, D box, and LBDspecific-signature motif are indicated by the bars above the alignment. The DNA-binding domain and the ligand-binding domain are underlined twice and once, respectively.

VD motor neurons (McIntire et al., 1993a,b, 1997; Jin et al., 1994). Although the expression of this genetic program explains the similarities that exist between the DD and VD motor neurons, it does not explain the synaptic patterns that distinguish the two motor neuron classes. UNC-55 is necessary for the production of the synaptic pattern that distinguishes the VD motor neurons from the DD motor neurons. Furthermore, loss of UNC-55 function causes the VD motor neurons to assume the synaptic pattern of the DD motor neurons (Walthall and Plunkett, 1995).

Table 1. Sequence similarity comparison of UNC-55 with hARP-1

\begin{tabular}{lllllll} 
Region & DBD & P Box & D Box & LBD & LBD-SSM & Overall \\
\hline Percentage & 91 & 100 & 86 & 44 & 90 & 50 \\
\hline
\end{tabular}



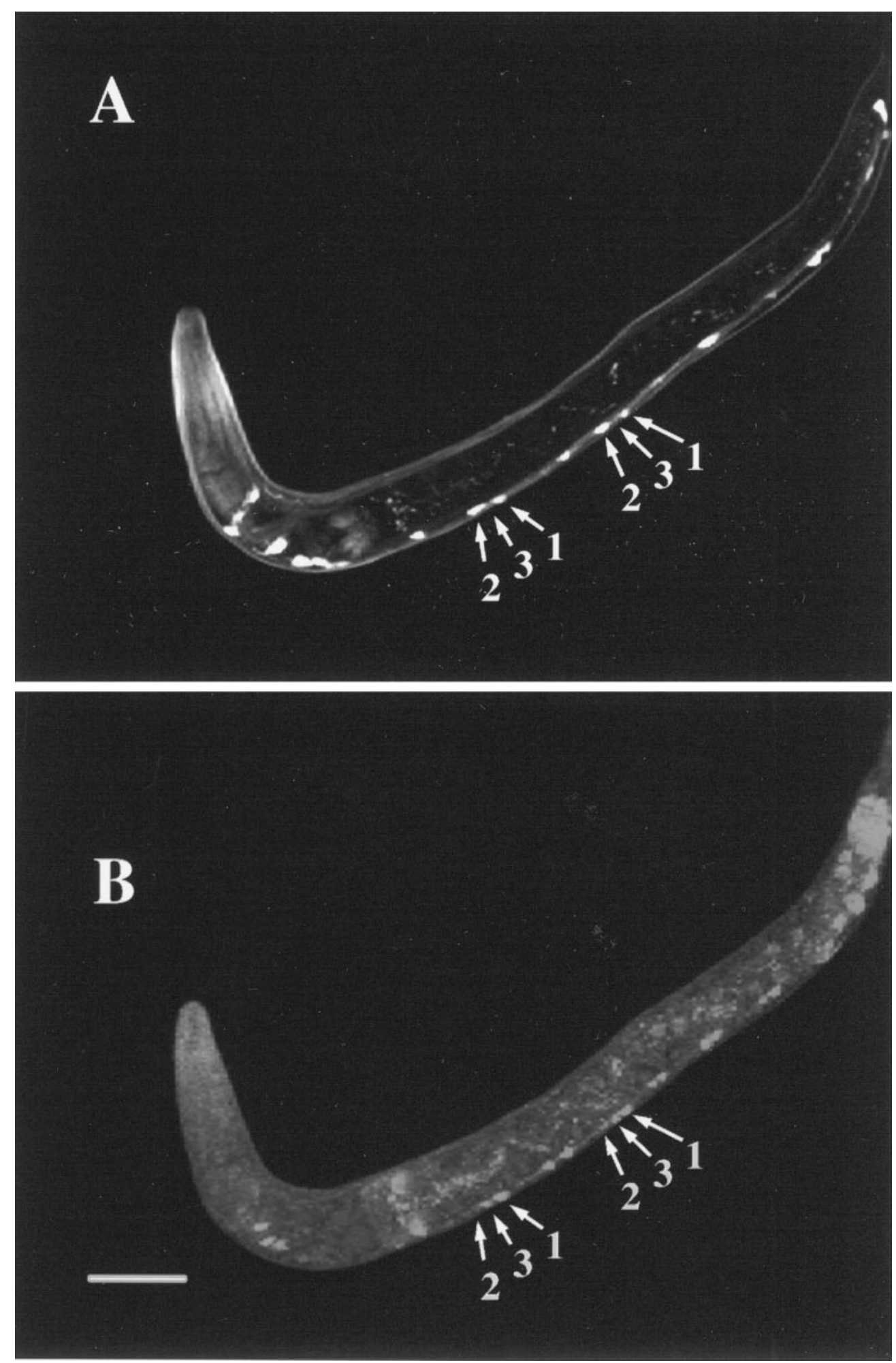

Figure 5. Simultaneous expression of the neurotransmitter GABA $(A)$ and an unc-55-gfp reporter $(B)$ in the nervous system of an L2 Stage $C$. elegans. 1, Cell bodies that colocalize GABA and the unc-55-gfp reporter; these are the VD motor neurons. 2, Locations of cell bodies that express GABA but not the unc-55-gfp reporter; these are the DD motor neurons. 3, Locations of cell bodies that express the unc-55-gfp reporter but not GABA; these have been identified tentatively as AS motor neurons. Criteria for motor neuron identification are described in Results. Anterior is to the left. Scale bar, $20 \mu \mathrm{m}$.

\section{UNC-55 is a member of the nuclear hormone receptor superfamily}

The amino acid sequence of UNC-55 shows a high degree of similarity to the COUP-TF family members, which are also known as orphan nuclear hormone receptors because ligands either have not been identified or possibly do not exist (Fig. 4). Recently, several reports have described roles for COUP-TFs in neural differentiation in zebrafish, Drosophila, and mice (Mlodzik 
et al., 1990; Fjose et al., 1993; Pereira et al., 1995). UNC-55 is currently the only member of the COUP-TF family found in $C$. elegans. Mutations in the Drosophila homolog seven-up ( $s v p$ ) cause photoreceptor cells R1, R3, R4, and R6 to adopt the fate of R7 cells (Mlodzik et al., 1990). This homeotic transformation in the photoreceptor cells is reminiscent of that of the VD motor neuron transformation observed in unc-55 mutants.

The COUP-TFs and SVP family members generally act as transcriptional suppressors (for review, see Mangelsdorf and Evans, 1995). This has been well characterized in SVP, which forms an inactive heterodimer with the ecdysone receptor. This reduction of functional ecdysone receptors leads to the activation of an alternative downstream promoter (Zelhof et al., 1995). This repression was recently characterized at the amino acid sequence level. A specific signature motif [(F/W)AK $X X X X \mathrm{~F} X X \mathrm{~L} X X X$ $\mathrm{DQ} X X \mathrm{LL}]$ is found within the LBD that is critical for the repressor activity (Downes et al., 1996). The sequence similarity among UNC-55, mammalian COUP-TFs, and SVP receptors in the LBD is less than that observed in the DBD. However, UNC-55 does share a number of critical amino acids with the LBD-specific signature motif (Fig. 4, position 164-181). The major difference is the number of amino acids between $\mathrm{K} / \mathrm{R}$ and $\mathrm{L}$ (Fig. 4, position 166-172), a region that is predicted to be a loop between two $\alpha$ helices (Bourguet et al., 1995). The variation in certain critical amino acids in the LBD may indicate that the amino acids in the loop are not important or that UNC-55 represents a new subclass in the group of COUP-TFs and SVP receptors.

\section{Spatial and temporal expression of an unc-55-gfp reporter}

The analysis of the unc-55-gfp expression in the ventral nerve cord revealed relatively tight spatial and temporal regulation. Expression was limited to the VD and probably the AS motor neurons during the L2 and L3 developmental stages (Fig. 5). Importantly, no unc-55-gfp staining was observed in the DD motor neurons during these developmenal stages. The expression of unc-55-gfp in a second set of ventral cord motor neurons was unexpected. We have tentatively identified this second class as the AS motor neurons. Lineally the 12 AS motor neurons are the anterior sisters of the VD motor neurons (Sulston and Horvitz, 1977). Of the five classes of postembryonic motor neurons, only VD and AS have processes in the dorsal and ventral nerve cord (White et al., 1986). Of the motor neurons involved in locomotion, the least is known about the role of the AS motor neurons. Their synaptic inputs and outputs are very similar to an embryonic class of motor neuron, the DAs. Like the DAs they are thought to be dorsal excitators involved in backward movement, but no attempt to analyze their role using genetic or surgical manipulations has been reported.

The tentative identification of AS motor neurons as expressing unc-55 impacts on a number of areas regarding the analysis of the uncoordinated phenotype and the role of unc-55 during development. The ventral coiling phenotype could be explained by a combination of changes associated with unc-55 mutations: the reduction of ventral inhibition and the addition of dorsal inhibition associated with the transformation of the VD motor neurons and the reduction of dorsal excitation that may result from compromising the AS motor neurons. Whether unc-55 is necessary for the differentiation and or function of the AS motor neurons remains to be determined.

\section{Synaptic respecification in the DD motor neurons}

In newly hatched animals the six embryonic DD motor neurons receive dorsal input and innervate ventral muscles, a synaptic pattern that is identical to the adult VD motor neurons. During the molt between the L1 and L2 stages when the VD motor neurons form their synapses, the DD motor neurons respecify their synapses and assume their adult innervation pattern (White et al., 1978). Thus both D motor neuron classes undergo synaptogenesis as the animal completes its first postembryonic molt, a period when the animal is immobile. This remodeling of the inhibitory neural circuits resembles the changes in identified neurons of certain holometabolous insects during metamorphosis (Levine, 1989). The insect molt is regulated by ecdysone, via the ecdysone receptor, which is also a member of the nuclear hormone receptor gene family (Koelle et al., 1991). The reorganization of the inhibitory neural circuits coincides with the first molt in $C$. elegans. The identification of a nuclear hormone receptor (UNC-55) that is in the same gene family as a number of Drosophila receptors that are involved in neural differentiation and are responsive to ecdysone is particularly intriguing (Tsai and O'Malley, 1994; Urness and Thummel, 1995) (for review, see Thummel, 1995). Although the hormone that initiates molting in C. elegans has not been identified, it is conceivable that UNC-55 in the VD motor neurons is activated by a hormone in conjunction with the molt cycle. Activation of these receptors could stabilize the synaptic pattern of the VD motor neurons by suppressing a response to a general (hormonal) signal that instructs the DD motor neurons to respecify their synaptic pattern and establish ventral inputs and dorsal outputs. In the absence of UNC-55 the VD motor neurons respond to the molt signal by default and form a synaptic pattern that is identical to the DD motor neurons. Specifically, we propose that activated UNC-55 receptors modify the expression of the common $\mathrm{D}$ motor neuron genetic program so that in the VD motor neurons proteins targeted for the presynaptic and postsynaptic processes are not redirected, whereas similar proteins in the DD motor neurons are redirected, thereby creating the synaptic pattern that distinguishes the two related classes of motor neurons.

In conclusion, the ability to integrate cellular, genetic, and molecular approaches with behavior in $C$. elegans has made it possible to identify and characterize a transcription factor that is necessary for establishing a synaptic pattern that distinguishes two functionally related classes of motor neurons from one another. We expect that the genes regulated by UNC-55 maintain the VD motor neuron synaptic pattern by establishing the polarity of the VD motor neuron cytoskeleton. The ubiquitous phylogenetic distribution of members of the nuclear hormone receptor gene family suggests that this has continued to be an important strategy for generating synaptic diversity as nervous systems have become more complex.

\section{REFERENCES}

Blumenthal T (1995) Trans-splicing and polycistronic transcription in Caenorhabditis elegans. Trends Genet 11:132-136.

Bourguet W, Ruff M, Chambon P, Gronemeter H, Moras D (1995) Crystal structure of the ligand-binding domain of the human nuclear receptor RXR-a. Nature 375:377-382.

Brenner S (1974) The genetics of Caenorhabditis elegans. Genetics 77:71-94.

Burke L, Downes M, Carozzi A, Giguere V, Muscat GE (1996) Transcriptional repression by the orphan steroid receptor RVR/Rev-erb beta is dependent on the signature motif and helix 5 in the $\mathrm{E}$ region: functional evidence for a biological role of RVR in myogenesis. Nucleic Acids Res 24:3481-3489. 
Chalfie M, Au M (1989) Genetic control of differentiation of the Caenorhabditis elegans touch receptor neurons. Science 243:1027-1033.

Chalfie M, Thomson JN, Sulston JE (1983) Induction of neuronal branching in Caenorhabditis elegans. Science 221:61-63.

Chalfie M, Sulston JE, White JG, Southgate E, Thomson JN, Brenner S (1985) The neurocircuit for touch sensitivity in Caenorhabditis elegans. J Neurosci 5:956-964.

Chalfie M, Tu Y, Euskirenchen G, Ward W, Prasher D (1994) Green fluorescent protein as a marker for gene expression. Science 263:802-805.

Collins J, Saari B, Anderson P (1987) Activation of a transposable element in the germ line but not the soma of Caenorhabditis elegans. Nature 328:726-728.

Downes M, Burke L, Muscat GE (1996) Transcriptional repression by $\mathrm{REv}$-erbA alpha is dependent on the signature motif and helix 5 in the ligand-binding domain: silencing does not involve an interaction with N-CoR. Nucleic Acids Res 24:3490-3498.

Eide D, Anderson P (1988) Insertion and excision of Caenorhabditis elegans transposable element Tc1. Mol Cell Biol 8:737-746.

Emmons SW, Klass MR, Hirsh D (1979) Analysis of the constancy of DNA sequences during development and evolution of the nematode Caenorhabditis elegans. Proc Natl Acad Sci USA 76:1333-1337.

Fjose A, Nornes S, Weber U, Mlodzik M (1993) Functional conservation of vertebrate seven-up related genes in neurogenesis and eye development. EMBO J 12:1403-1414.

Glass CK (1994) Differential recognition of target genes by nuclear receptor monomers, dimers and heterodimers. Endocr Rev 15:391-407.

Hedgecock E, Culotti J, Hall D (1990) The unc-5, unc-6, and unc-40 gene guide circumferential migration of pioneer axons and mesodermal cells on the epidermis in C. elegans. Neuron 2:61-85.

Jin YS, Hoskins R, Horvitz HR (1994) Control of type-D GABAergic neuron differentiation by $\mathrm{C}$. elegans UNC-30 homeodomain protein. Nature 372:780-783.

Jones AR, Schedl T (1995) Mutations in gld-1, a female germ cellspecific tumor suppressor gene in Caenorhabditis elegans, affect a conserved domain also found in Scr-associated protein Sam68. Genes Dev 9:1491-1504.

Jonk LJ, Jonge ME, Pals CE, Wissink S, Vervaart JM, Schoorlemmer J, Fruijer W (1994) Cloning and expression during development of three murine members of the COUP family of nuclear orphan receptors. Mech Dev 48:81-97.

Koelle MR, Talbot WS, Segraves WA, Bender MT, Cherbas P, Hogness DS (1991) The Drosophila EcR gene encodes an ecdysone receptor, a new member of the steroid receptor superfamily. Cell 67:59-77.

Kozak M (1991) Structural features in eukaryotic mRNAs that modulate the initiation of translation. J Biol Chem 266:19867-19870.

Kramer JM, French RP, Park EC, Johnson JJ (1990) The Caenorhabditis elegans rol-6 gene, which interacts with the sqt-1 collagen gene to determine organismal morphology, encodes a collagen. Mol Cell Biol 10:2081-2089.

Krause M, Hirsh D (1987) Trans-spliced leader sequence on actin mRNA in C. elegans. Cell 49:753-761.

Ladias JA, Karathanasis SK (1991) Regulation of the Apolipoprotein AI gene by ARP-1, a novel member of the steroid receptor superfamily. Science 251:561-565.

Levine RB (1989) Expansion of the central arborizations of persistent sensory neurons during insect metamorphosis: the role of the steroid hormone, 20-hydroxyecdysone. J Neurosci 9:1045-1054.

Mangelsdorf DJ, Evans RM (1995) The RXR heterodimers and orphan receptors. Cell 83:841-850.

McIntire SL, Garriga G, Horvitz R (1992) Genes necessary for directed axonal elongation or fasciculation in C. elegans. Neuron 8:307-322.
McIntire SL, Jorgensen EM, Horvitz HR (1993a) Genes required for GABA function in Caenorhabditis elegans. Nature 364:334-337.

McIntire SL, Jorgensen EM, Horvitz HR (1993b) The GABAergic nervous system of Caenorhabditis elegans. Nature 364:337-341.

McIntire SL, Reimer RJ, Schuske K, Edwards RH, Jorgensen EM (1997) Identification and characterization of the vesicular GABA transporter. Nature 389:870-876.

Mello CC, Kramer JM, Stinchcomb D, Ambros V (1991) Efficient gene transfer in C. elegans: extrachromosomal maintenance and integration of transforming sequences. EMBO J 10:3959-3970.

Mlodzik M, Hiromi Y, Weber U, Goodman CS, Rubin GM (1990) The Drosophila seven-up gene, a member of the steroid receptor gene superfamily, controls photoreceptor cell fates. Cell 60:211-224.

O'Leary DD, Terashima T (1988) Cortical axons branch to multiple subcortical tragets by interstitial axon budding: implication for target recognition and "waiting periods." Neuron 1:901-910.

Pereira FA, Qiu Y, Tsai MJ, Tsai SY (1995) Chicken ovalbumin upstream promoter transcription factor (COUP-TF): expression during mouse embryogenesis. J Steroid Biochem Mol Biol 53:503-508.

Sambrook J, Fritsch EF, Maniatis T (1989) Molecular cloning: a laboratory manual. Cold Spring Habor, NY: Cold Spring Habor Laboratory.

Sulston J, Horvitz HR (1977) Postembryonic cell lineages of the nematode Caenorhabditis elegans. Dev Biol 56:110-156.

Thummel CS (1995) From embryogenesis to metamorphosis: the regulation and function of Drosophila nuclear receptor superfamily members. Cell 83:871-877.

Truss M, Beato M (1993) Steroid hormone receptors: interaction with DNA and transcription factors. Endocr Rev 14:459-479.

Tsai MJ, O'Malley BW (1994) Molecular mechanisms of action of steroid/thyroid receptor superfamily members. Annu Rev Biochem 63:451-486.

Umesono K, Evans RM (1989) Determinants of target gene specificity for steroid/thyroid hormone receptors. Cell 57:1139-1146.

Urness LD, Thummel CS (1995) Molecular analysis of a steroid-induced regulatory hierarchy: the Drosophila E74A protein directly regulates L71-6 transcriptions. EMBO J 14:6239-6246.

Walthall WW, Chalfie M (1988) Cell-cell interactions in the guidance of late-developing neurons in Caenorhabditis elegans. Science 239:643-645.

Walthall WW, Plunkett JA (1995) Genetic transformation of the synaptic pattern of a motoneuron class in Caenorhabditis elegans. J Neurosci 15:1035-1043.

Wang LH, Tsai SY, Cook RG, Beattie WG, Tsai MJ, O'Malley BW (1989) COUP transcription factor is a member of the steroid receptor superfamily. Nature 340:163-166.

Way JC, Chalfie M (1988) mec-3, a homeobox containing gene that specifies differentiation of the touch receptor neurons in C. elegans. Cell 54:5-16.

White JG, Alberston DG, Anness MAR (1978) Connectivity changes in a class of motoneuron during the development of a nematode. Nature 271:764-766.

White JG, Southgate E, Thomson JN, Brenner S (1986) The structure of the nervous system of the nematode Caenorhabditis elegans. Philos Trans R Soc Lond B Biol Sci 314:1-340.

Xue D, Tu Y, Chalfie M (1993) Cooperative interactions between the Caenorhabditis elegans homeoproteins UNC-86 and MEC-3. Science 261:1324-1328.

Zelhof AC, Yao T, Evans RM, McKeown M (1995) Seven-up inhibits ultraspiracle-based signaling pathways in vitro and in vivo. Mol Cell Biol 15:6736-6745. 